Sharif University of Technology
Scientia Iranica
SCIENTIA

Invited Paper

\title{
Evaluation of online techniques utilized for extracting the transformer transfer function
}

\author{
F. Nasirpour, M.H. Samimi*, and H. Mohseni \\ School of Electrical and Computer Engineering, University College of Engineering, University of Tehran, North Kargar St., Tehran,
} P.O. Box 14395-515, Iran.

Received 27 June 2019; accepted 8 September 2019

\section{KEYWORDS \\ Fault diagnosis; \\ Fourier transform; \\ Frequency response analysis; \\ Online diagnosis; \\ Power transformer monitoring; \\ Transfer function; \\ Wavelet transform.}

\begin{abstract}
Power transformers are of vital importance in power delivery and, therefore, different diagnostic techniques have been proposed for them. The Frequency Response Analysis (FRA) is an effective method that detects mechanical changes in transformer windings by extracting the transfer function. There are various approaches to obtaining the transfer function online, which is known as the online FRA technique. This paper compares these different mathematical approaches for obtaining the transfer function of a transformer. A comparison is carried out by defining an appropriate model for the transformer and applying these mathematical methods to it. The effect of other power network equipment on the transformer transfer function is also studied in this paper. The results of this contribution determine proper methods for the online FRA technique, which can be used in the transformer monitoring applications.
\end{abstract}

(C) 2019 Sharif University of Technology. All rights reserved.

\section{Introduction}

Power transformers are considered one of the most important equipment in the power system. They operate in various climates and are subject to different types of mechanical and electrical stresses. Hence, transformers can always encounter faults [1]. The power system may face outage problems that can result in a considerable costly impact on utilities if transformer faults are not detected in the early stage [2]. Therefore, it is vital to continuously evaluate transformers' conditions in order to diagnose problems that may occur. For this purpose, many different mechanical, electrical,

\footnotetext{
*. Corresponding author. Tel.: +9821 88011247;

Fax: +982188027766

E-mail addresses: Farzad.nasirpour@ut.ac.ir (F.

Nasirpour); m.h.samimi@ut.ac.ir (M.H. Samimi);

mohseni@ut.ac.ir (H. Mohseni)
}

doi: $10.24200 /$ sci.2019.53890.3473 chemical, and optical methods have been introduced and employed $[3,4]$.

Winding deformation, turn-to-turn short circuit, and insulation degradation are some types of transformer faults. One of the widely used techniques for detecting these faults is the Frequency Response Analysis (FRA), which consists of obtaining and analyzing the transfer function of the transformer in the desired bandwidth [5].

The FRA technique is mainly applied in the offline mode, in which the transformer should be taken out of service. According to the nature of the excitation signal, two measurement methods can be distinguished in the offline FRA technique: the Impulse Frequency Response Analysis (IFRA) and the Sweep Frequency Response Analysis (SFRA) [5,6]. In the IFRA, a lowvoltage impulse is applied to the winding as the excitation, and the transformer response is measured in the time domain. The input current, output voltage, and neutral current can be considered as the transformer 
response in the IFRA method. On the other hand, a sinusoidal signal with a fixed amplitude and varying frequencies is applied to the transformer in the SFRA technique, and the response is measured directly in the frequency domain. In this case, the transfer function is usually the ratio of the output voltage to the input voltage. A typical range of frequency sweeps is from $20 \mathrm{~Hz}$ to $2 \mathrm{MHz}$ [7].

The main drawback of the offline FRA technique is that the transformer has to be taken out of service, which may interrupt the energy delivery in the power network [8]. Furthermore, the external factors affecting the FRA can be different in two consecutive measurements since the interval between two measurements is several months or years. For instance, the possible temperature variation between different measurements can have a significant impact on the captured FRA trace [9]. The interval between consecutive tests also may cause changes in the transfer function because of the variation of the paper humidity. These unwanted errors and uncertainties yield sometimes in misunderstanding the transformer condition. By employing the online FRA technique, the aforementioned errors are dismissed since the FRA is captured more frequently, and the interval between measurements is significantly shortened.

Capturing the online FRA enjoys other benefits, too. By applying this method, there is no need to take the transformer out of service, which saves costs and time and is in accordance with the new requirements of the power system [10,11]. Moreover, the transformer is evaluated in shorter time frames, i.e., the transformer is monitored more appropriately. In the online FRA technique, the excitation signal can be power system transients caused by lightning and switching operations or a pulse that is manually injected to the transformer [12].

The assessment of the transformer condition by the FRA technique has been widely studied in the last decade. Various methods of transformer evaluation compared to FRA technique and also different mathematical approaches for obtaining the transfer function have been studied in the literature $[1,5,13,14]$. To implement the FRA by the injection of excitation signals, several practical methods and related issues were presented in the referenced studies [7,15-17]. A number of authors conducted further studies on faulttype classification, the FRA sensitivity, and temperature dependency of the FRA [18-20]. Gomez-Luna et al. presented a review of the current status and future trends of the online FRA technique [6].

Regardless of previous studies, there is no record of comparing the different methods for obtaining the online transfer function of the transformer. In other words, it is not yet specified which mathematical method can extract the online FRA trace of the transformer more appropriately. The focus of the current contribution in this study is to compare these methods and determine a proper mathematical tool for the online FRA.

In this paper, different methods presented in the literature for obtaining the online transfer function of transformer winding are compared, and the best method is identified. For this purpose, the transfer function obtained by the SFRA method is considered as the reference. The method that results in a trace more analogous to the SFRA trace is a better one. In addition to this comparison, the effect of other power network apparatuses on the transformer transfer function is studied. Accordingly, an attempt is made here to find out if the other existing equipment affects the captured trace of a transformer.

The rest of this paper is organized as follows. Section 2 discusses the mathematical approaches briefly. Section 3 describes the details of modeling a transformer winding for the purpose of investigating different online methods. Afterward, different methods for determining an online frequency response are studied in Section 4, and the corresponding results are presented. In Sections 5, the effect of power network equipment on an online transfer function is investigated. The conclusion is finally given in Section 6 .

\section{Theoretical background}

The online FRA technique is based on measuring the transfer function of the transformer winding. As mentioned before, different currents and voltages can be considered as the transformer transfer function. The first step for obtaining the transfer function is to measure these signals in a wide frequency range. For this purpose, a high-frequency signal can be applied to the transformer winding, and the corresponding response signal is measured in the time domain. The high-frequency signal can be a power system transient, e.g., lightning and switching phenomena.

Figure 1 shows a basic method for online transfer function measurement using network transients as the excitation signal. As can be seen, a transient signal reaches the transformer winding through the connected transmission lines. Upon receiving the signal, the signal itself and a response signal are measured by sensors and, then, processed by the signal processing unit. The method described in Figure 1 measures the input and output voltages using two metal foil pieces inserted near the bushing flange. These metal foil pieces act as a capacitive sensor for measuring the voltage signal. Similarly, by employing a high-frequency current transformer on the neutral lead, the neutral current can be captured as the response signal.

After measuring any of the described signals in the time domain, these signals are transferred to the 


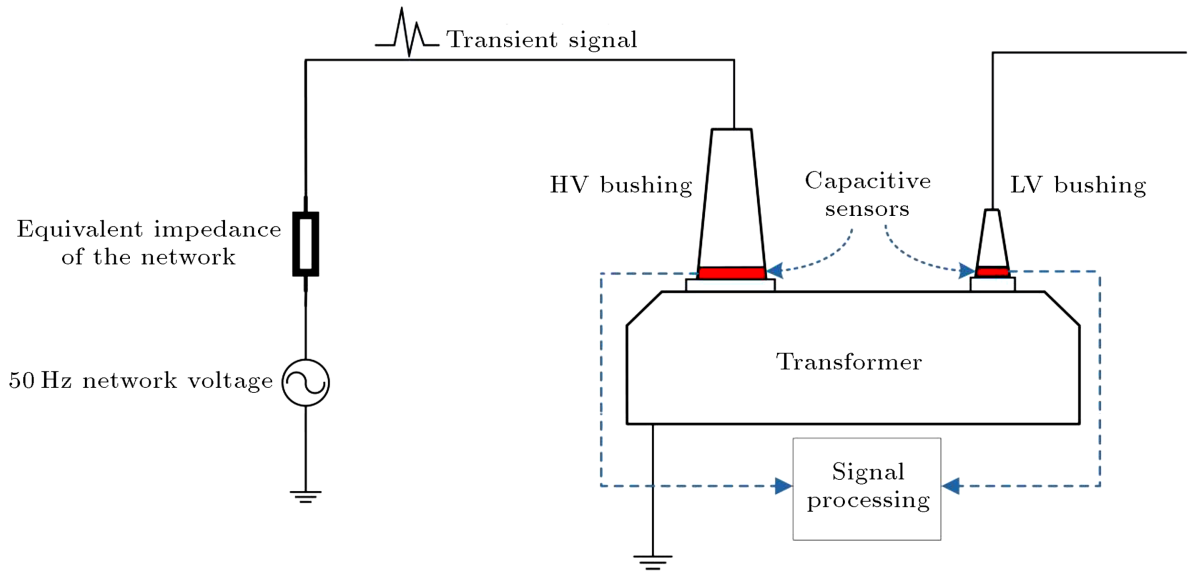

Figure 1. The basic circuit for measuring the online FRA.

frequency domain by utilizing mathematical methods. Different mathematical methods such as Fast Fourier Transform (FFT), Short Time Fourier Transform (STFT), and wavelet transform are used for achieving this goal in the literature $[3,9,10]$. In the following subsections, the basics of each method are discussed briefly. In the final stage, the signals transferred to the frequency domain are divided by each other to gather the intended transfer function.

\subsection{Fast Fourier Transform (FFT)}

The FFT is an algorithm for computing the Discrete Fourier Transform (DFT). The DFT is defined as follows [15]:

$$
X_{k}=\sum_{n=0}^{N-1} x[n] e^{(-i 2 \pi k n / N)} \quad k=0, \ldots \ldots, N-1,
$$

where $x[n]$ is the discrete input signal, $N$ is the total number of samples, and $k$ is the desired frequency.

After measuring the signals in the time domain, the FFT of the excitation and the response signals are measured through Eq. (1):

$$
\begin{aligned}
& V_{\text {in }}(f)=F F T\left(v_{\text {in }}(t)\right), \\
& V_{\text {out }}(f)=F F T\left(v_{\text {out }}(t)\right),
\end{aligned}
$$

where $v_{\text {in }}$ and $v_{\text {out }}$ are the discrete signals in the time domain, and $V_{\text {in }}$ and $V_{\text {out }}$ are the signals transferred to the frequency domain. The transfer function has complex values; however, the evaluation of frequency response is usually performed on its magnitude. In this paper, the transfer function is defined similarly as the ratio of the response magnitude to the excitation magnitude as follows:

$$
T F_{\text {IFRA }}=20 \log \left(\frac{\left|V_{\text {out }}(f)\right|}{\left|V_{\text {in }}(f)\right|}\right),
$$

where $T F$ is the transfer function. The transfer function is normally displayed in a log-log plot.

\subsection{Short Time Fourier Transform (STFT)}

The discrete STFT of function $x[n]$ is expressed as in the following equation [13]:

$$
\begin{aligned}
\operatorname{STFT}\{x[n]\}(m, \omega) & \equiv X(m, \omega) \\
& =\sum_{n=-\infty}^{\infty} x[n] \omega[n-m] e^{-j \omega n},
\end{aligned}
$$

where $\omega[m]$ is the window function that is nonzero for only a short period of time. In the STFT method, the window function divides the signal into equal, shorter segments and, then, the Fourier transform of each segment is computed. The length of each segment equals that of the window function. The Blackman window function, as shown in Figure 2, is used in this paper for dividing the input signal. It is noteworthy that the Blackman function is one of the most used window functions in the STFT method.

STFT transfers a signal from the time domain to the time-frequency domain; however, in order to obtain the transfer function, frequency domain data are required. Here, the transfer function is defined as in Eq. (6), which provides the frequency domain information of the signal.



Figure 2. Blackman window. 


$$
T F_{\text {IFRA }}=\frac{\sum_{m=-\infty}^{\infty} \sum_{n=-\infty}^{\infty} V_{\text {out }}[n] \omega[n-m] e^{-j \omega n}}{\sum_{m=-\infty}^{\infty} \sum_{n=-\infty}^{\infty} V_{\text {in }}[n] \omega[n-m] e^{-j \omega n}}
$$

where $V_{\text {out }}$ and $V_{\text {in }}$ are STFT of the response and excitation signals, respectively.

\subsection{Wavelet transform}

The continuous wavelet transform of function $x(t)$ is defined as follows [5]:

$$
X(a, b)=\frac{1}{|a|^{1 / 2}} \int_{-\infty}^{\infty} x(t) \bar{\psi}\left(\frac{t-b}{a}\right) d t,
$$

where $a$ is the scale, $b$ is the translational value, and $\psi$ is the mother wavelet. In this paper, Morlet is used as the mother wavelet. Unlike the STFT, the window function has variable lengths in the wavelet transform, which is defined by the parameter $a$.

The wavelet transform also transfers signals from the time domain to the time-scale domain. Therefore, Eq. (8) should be used to obtain information in the scale domain.

$$
F(a)=\sqrt{\frac{\sum_{\tau}\left(C_{V \text { out }}(\tau, a)\right)^{2}}{\sum_{\tau}\left(C_{V \text { in }}(\tau, a)\right)^{2}},}
$$

where $C_{V \text { out }}$ and $C_{V \text { in }}$ are the continuous wavelet transforms of the response and the excitation signals, respectively. There must be a relationship between the scale and frequency so that one can express the frequency domain information of the signal from the scale domain. Although there is no exact relation between these two, the following approximation can be applied [5]:

$$
f_{a}=\frac{f_{c}}{a \cdot \Delta},
$$

where $f_{c}$ is the center frequency of a wavelet in $\mathrm{Hz}$, and $\Delta$ is the sampling period. Consequently, the transfer function can be obtained through Eqs. (8) and (9).

\section{Modeling the transformer windings}

In the previous section, the details of the three mathematical methods for obtaining the online transfer function have been described. In order to examine these methods, a winding with the known transfer function is required. In this contribution, a high-frequency model of the winding is considered as the test case. All methods with regard to this model are examined whose details are described in this section [21].

In the utilized model, the winding is divided into several winding units, where each unit, as shown in
Figure 3, consists of $R, L, C$, and $M$ that represent the electrical characteristics of the unit. The winding unit can be a disk, two disks or several turns. This model is valid for the frequency range of a few $\mathrm{kHz}$ up to approximately $1 \mathrm{MHz}[21]$.

The elements of this model can be calculated by analytical formulas [21]. In this paper, an HV winding with 60 discs, 11 turns in each disk with a 20-turn helical LV winding, and 12 parallel conductors in each turn is modeled. Each disk of the HV winding is considered as an HV winding unit. Similarly, each LV winding turn is considered as the LV winding unit. Figure 4 shows the windings diagram and the corresponding

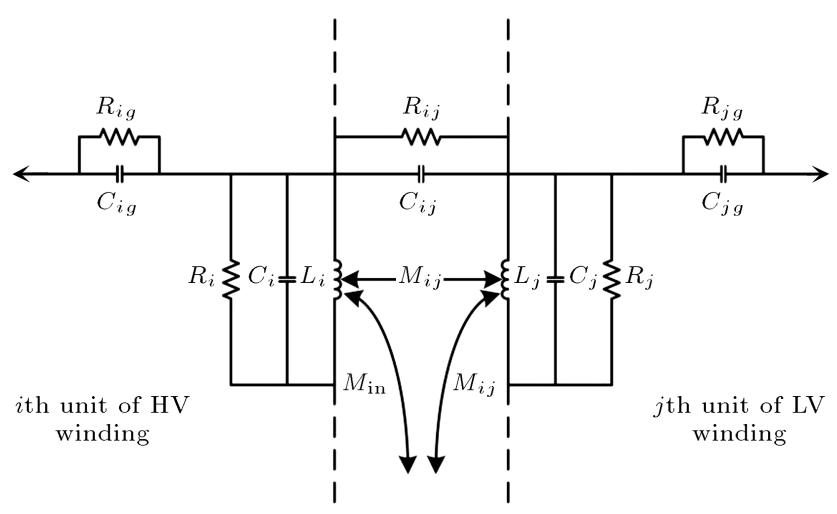

Figure 3. The detailed model of transformer windings based on mutual inductance.



Figure 4. The diagram of the modeled winding. The dimensions are in $\mathrm{cm}$. 
dimensions needed for calculating the model parameters. All of the elements depend on the geometrical dimensions of winding units and transformer; thus, any change in the geometrical dimensions influences the values of these elements and, therefore, the frequency response changes. In the following paragraphs, the calculation of these parameters is briefly explained:

- Self and mutual inductance: Maxwell's equations can be solved to calculate the self and mutual inductances between the winding units. The mutual inductance between two loops shown in Figure 5 can be calculated using Eq. (10):

$$
M_{12}=\frac{\mu_{0}}{4 \pi} \oint_{C_{1}} \oint_{C_{2}} \frac{d \vec{s}_{1} \cdot d \vec{s}_{2}}{R_{12}}
$$

where $\mu_{0}$ is the permittivity of the vacuum, and the rest of the parameters are depicted in Figure 5. This equation can be rewritten as follows:

$$
\begin{aligned}
M_{12} & =\frac{\mu_{0}}{4 \pi} \int_{0}^{2 \pi} \int_{0}^{2 \pi} \frac{r_{1}\left(\alpha_{1}\right) \cdot r_{2}\left(\alpha_{2}\right) \cdot \cos \left(\alpha_{2}-\alpha_{1}\right)}{R_{12}\left(\alpha_{1}, \alpha_{2}\right)} . \\
& d \alpha_{1} \cdot d \alpha_{2} .
\end{aligned}
$$

For un-deformed turns, the analysis of Eq. (11) results in the closed Eq. (12):

$$
M_{12}=\frac{2 \mu_{0} \sqrt{r_{1} r_{2}}}{\sqrt{k^{\prime}}} \cdot\left[K\left(k^{\prime}\right)-E\left(k^{\prime}\right)\right]
$$

where:

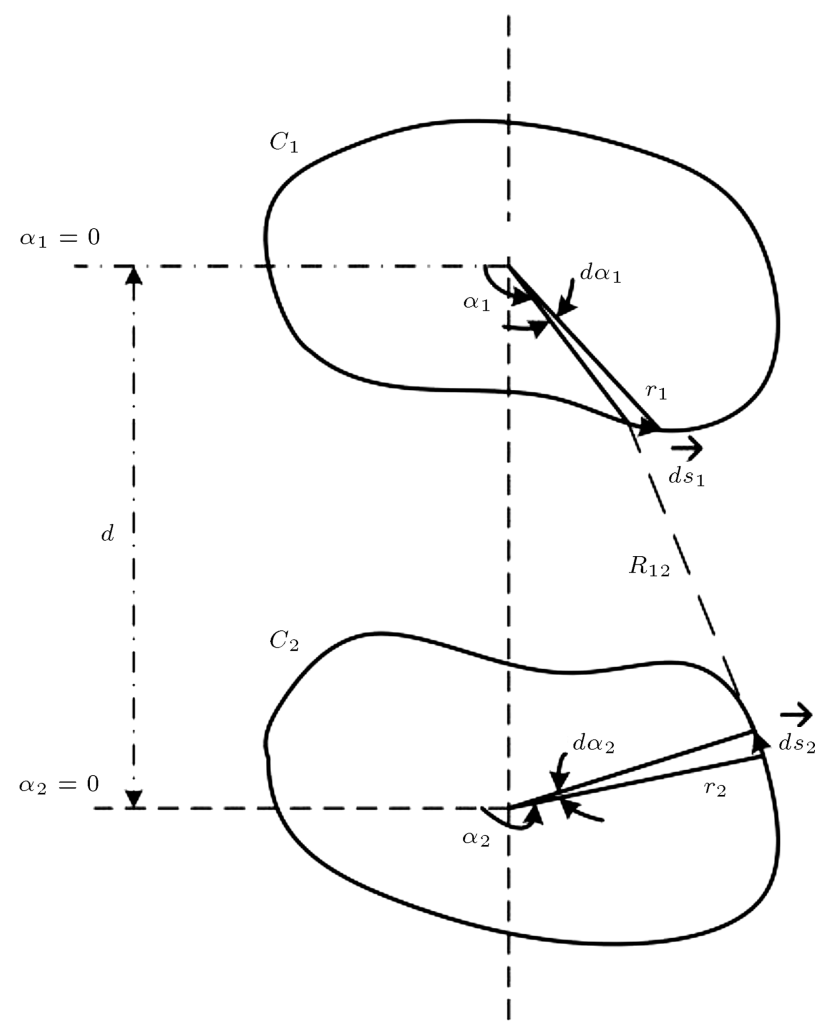

Figure 5. Two parallel conducting loops [21].

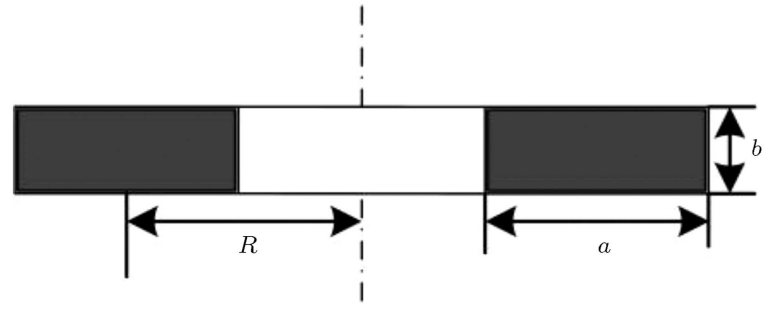

Figure 6. Parameter definition of one-single turn [21].

$$
k^{\prime}=\frac{1-\sqrt{1-k^{2}}}{1+\sqrt{1-k^{2}}}, \quad k=\sqrt{\frac{4 r_{1} r_{2}}{\left(r_{1}+r_{2}\right)^{2}+d^{2}}}
$$

$K\left(k^{\prime}\right)$ and $E\left(k^{\prime}\right)$ are the complete elliptic integrals of the first and second kinds, respectively.

For an un-deformed turn, shown in Figure 6, self-inductance can be calculated using Eq. (14):

$$
L_{i}=\mu_{0} R\left(\ln \frac{8 R}{G M D}-2\right),
$$

where:

$$
\begin{aligned}
\ln \frac{G M D}{\sqrt{a^{2}+b^{2}}}= & \frac{2 b}{3 a} \tan ^{-1} \frac{a}{b}+\frac{2 a}{3 b} \tan ^{-1} \frac{b}{a} \\
& -\frac{b^{2}}{12 a^{2}} \ln \left(1+\frac{a^{2}}{b^{2}}\right) \\
& -\frac{a^{2}}{12 b^{2}} \ln \left(1+\frac{b^{2}}{a^{2}}\right)-\frac{25}{12} .
\end{aligned}
$$

The parameters of the single turn needed for calculating Eq. (15) are illustrated in Figure 6;

- Capacitance: $C_{i g}$ and $C_{j g}$ represent the capacitance between the individual winding unit and the earth, which can be the transformer tank or the core. $C_{i j}$ represents the capacitance between two windings ( $\mathrm{HV}$ and LV). These capacitances can be calculated based on the homogenous distribution of the electric field.

$C_{i}$ and $C_{j}$ represent the energy stored between the turns of the winding unit and can be calculated similar to $C_{i g}$, assuming that there is a linear voltage distribution along with the winding unit [21].

- Resistance: $R_{i g}, R_{j g}, R_{i}, R_{j}$, and $R_{i j}$ represent the dielectric losses of the dielectric system between winding units and also between windings and the tank. All of the resistances are frequency dependent since the dielectric loss changes with frequency. Compared to dielectric resistances, the conductor resistance has insignificant effect on damping mechanisms and, thus, is ignored for the sake of simplicity.

\section{Comparing the mathematical methods}

In this section, the mathematical methods are compared with each other. The winding model described 
in Section 3 is utilized as the test object for examining the methods.

\subsection{Examining the mathematical methods in the offline mode}

In the first step, the methods are investigated in the offline mode. For this purpose, a pulse according to Eq. (16) is injected into the transformer $\mathrm{HV}$ winding, and the input current is measured as the response. Figure 7 shows these signals in the time domain for the modeled winding. As can be seen, the excitation signal is very short in the time frame; however, the response signal continues to oscillate in a wider time frame. The sampling frequency is $10 \mathrm{MHz}$ and the sampling duration should be greater than $10 \mathrm{~ms}$. After recording these traces, the aforementioned mathematical methods are applied to both signals to obtain the frequency response.

$$
V_{\text {pulse }}(t)=10 e^{\frac{-18.4207}{10^{-5}}\left(t-5 \times 10^{-6}\right)^{2}} .
$$

In order to determine the best method, the transfer function obtained from the SFRA method is considered as the reference. In other words, all obtained transfer functions are compared with the SFRA, and the method that results in the trace most analogous to the SFRA trace is chosen as the proper method. A similar approach is carried out in the online mode, too.

For capturing the SFRA trace of the winding, a procedure similar to the experimental setup is employed. A sinusoidal voltage source is used as an excitation signal. The frequency of this signal is swept in the frequency range of $10 \mathrm{kHz}$ to $1.5 \mathrm{MHz}$ while the amplitude is kept constant equal to $1 \mathrm{~V}$. In each frequency point, the excitation and response signal are measured in the time domain. The input signal has a single frequency. The response signal is also filtered based on the same frequency and, therefore, two sinusoidal signals with the same frequency are derived.


Figure 7. The excitation and the response signals of the modeled winding. The excitation signal is a voltage impulse, and the response signal is the input current.

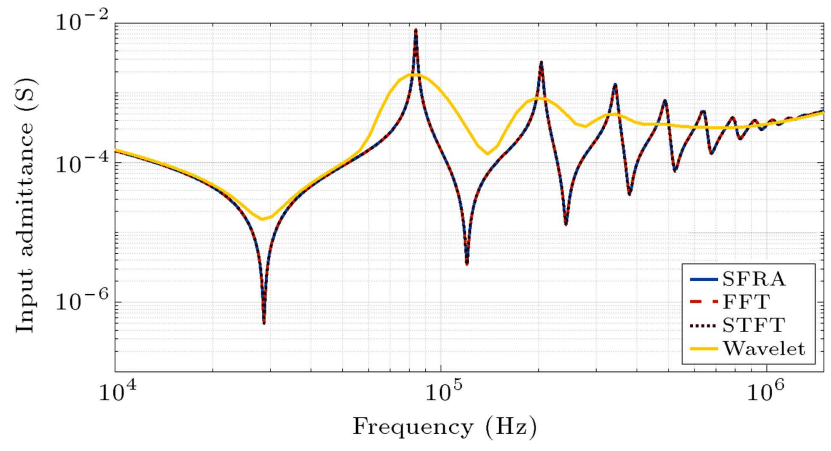

Figure 8. Comparison of the offline transfer function trace captured by different methods and the SFRA trace.

The transfer function is then obtained by dividing the magnitude of the response signal by the magnitude of the excitation signal at each point. In this case, the input current of the $\mathrm{HV}$ winding is considered as the response because the same signal is employed for examining the mathematical methods.

Figure 8 shows the frequency response traces obtained using FFT, STFT, and the wavelet transform in comparison with the SFRA method. As can be inferred from Figure 8, except for the wavelet transform, the other two methods are in complete agreement with the SFRA trace. Therefore, it can be concluded that the wavelet is not a suitable method since it does not give a good answer even in the offline mode, where the $50 \mathrm{~Hz}$ signal is missing. Nevertheless, all three methods are examined in the online mode in the next section.

\subsection{Examining the mathematical methods in the online mode}

For this assessment, the transformer is connected to the $50 \mathrm{~Hz}$ ac voltage. The transient pulse mentioned in the previous section is then superimposed onto it. Therefore, the measured signals have both a considerable magnitude in the $50 \mathrm{~Hz}$ and high-frequency contents. It is possible to perform the methods directly on the measured signal. The other way is to filter the $50 \mathrm{~Hz}$ signal first and, then, apply the mathematical methods.

In order to eliminate the $50 \mathrm{~Hz}$ frequency component, a typical Butterworth high-pass filter of the 2nd order can be used. This filter can be implemented digitally. The transfer function of the utilized filter in the Laplace domain is as follows:

$$
H(s)=0.99^{2}\left(\frac{s^{2}-2 s+1}{s^{2}-1.9 s+0.99}\right)^{2} .
$$

It is noteworthy that the $3 \mathrm{~dB}$ cut-off frequency of this filter equals $100 \mathrm{~Hz}$ and, therefore, the filter does not influence the higher frequency content of the signal. Accordingly, the filter does not change the phase information of the response in case that the phase response is also required for the interpretation. 


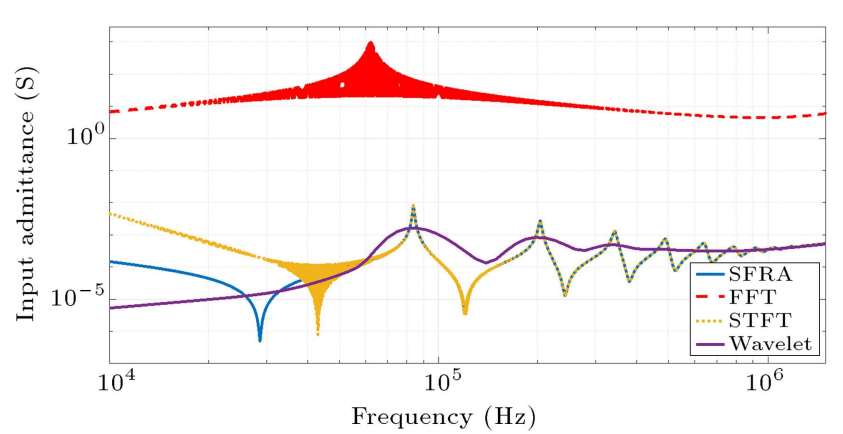

Figure 9. The online transfer function trace captured by different methods without filtering the $50 \mathrm{~Hz}$ frequency component.

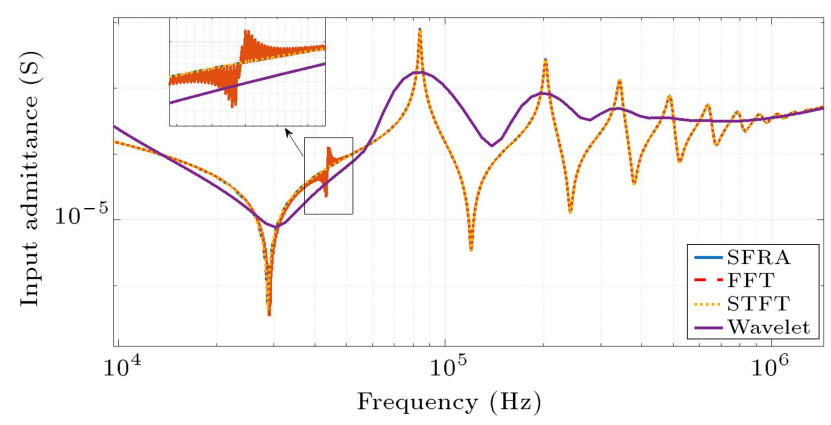

Figure 10. The online transfer function trace captured by different methods by filtering the $50 \mathrm{~Hz}$ frequency component.

Figures 9 and 10 demonstrate the output of various methods with and without filtering the $50 \mathrm{~Hz}$ frequency component, respectively. In the online mode and without filtering the $50 \mathrm{~Hz}$ frequency component, FFT and wavelet methods have poor agreement with the SFRA in comparison with the STFT method. Even if the $50 \mathrm{~Hz}$ frequency component is filtered, the FFT method shows some fluctuations that may lead to misunderstanding the transformer condition. This fluctuation is magnified in the subset of Figure 10. It is noteworthy that the FFT method shows good agreement in the offline mode, though it demonstrates a weaker performance than the STFT method in the online mode. Therefore, it can be concluded that the STFT method is better than FFT and wavelet methods for obtaining the frequency response.

Hitherto, the effect of different mathematical methods on the frequency response has been studied, and it has been demonstrated that STFT has the best performance in obtaining the frequency response. It should be noted that, until now, the input current of the $\mathrm{HV}$ winding has been considered as the response. There are also other responses that can be examined. The voltage of the secondary side is now considered as the response.

Figure 11 shows the voltage transfer function captured by different methods along with the voltage SFRA trace as the reference. It is evident that even in

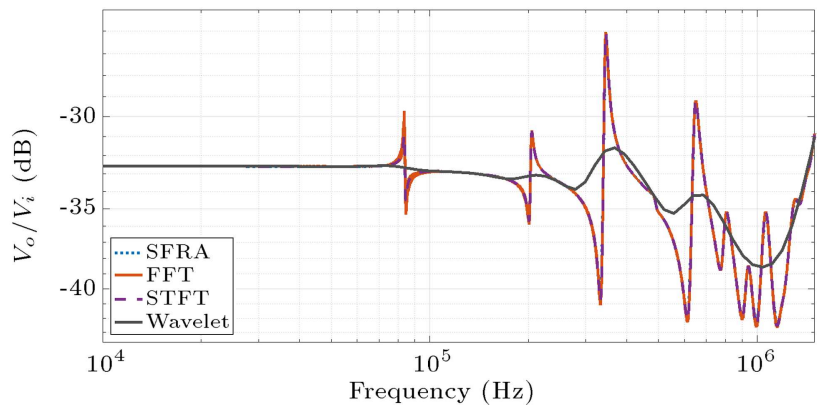

Figure 11. The transfer function without filtering $50 \mathrm{~Hz}$ frequency component.

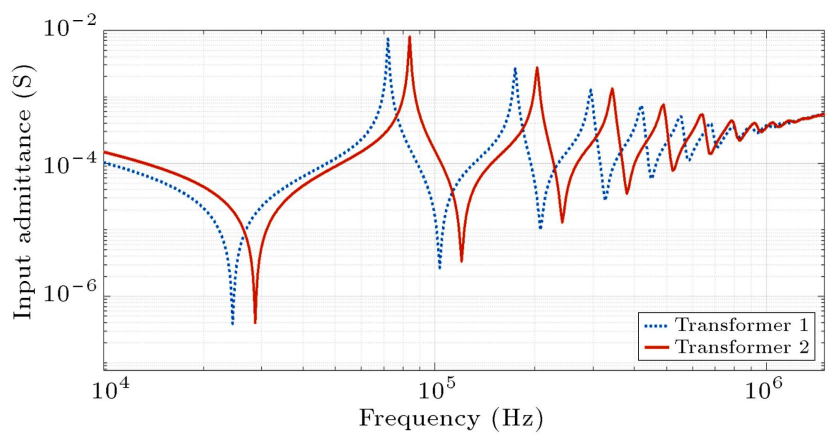

Figure 12. The frequency response (SFRA) of the two transformers in parallel.

the presence of the $50 \mathrm{~Hz}$ frequency component, both SFTF and FFT methods have good agreement with the SFRA trace. In other words, both STFT and FFT express good responses in the voltage mode, though the FFT is weaker when the current is the response signal. In summary, it can be concluded that STFT works appropriately in both current and voltage waveforms and, therefore, is a suitable method for the online FRA.

\section{Effect of adjacent equipment on the online transfer function of a transformer}

Investigating the effect of the power system on the online frequency response of a transformer is one of the challenging issues that has been always a matter of concern. In order to investigate such effects, two parallel transformers with different frequency responses are assumed here. The differences between these two frequency responses are achieved by varying the dimensions of the mentioned transformer and the modeling elements accordingly. The frequency responses of these two transformers, obtained in the offline mode, are presented in Figure 12. These traces are in fact the SFRA of these transformers and the reference to determine whether the measured online transfer function still corresponds with the target transformer.

These two parallel no-load transformers are connected via a $60 \mathrm{~km}$ transmission line to a $50 \mathrm{~Hz}$ source modeling the rest of the power system. A lightning 




Figure 13. The schematic connection of two parallel transformers.

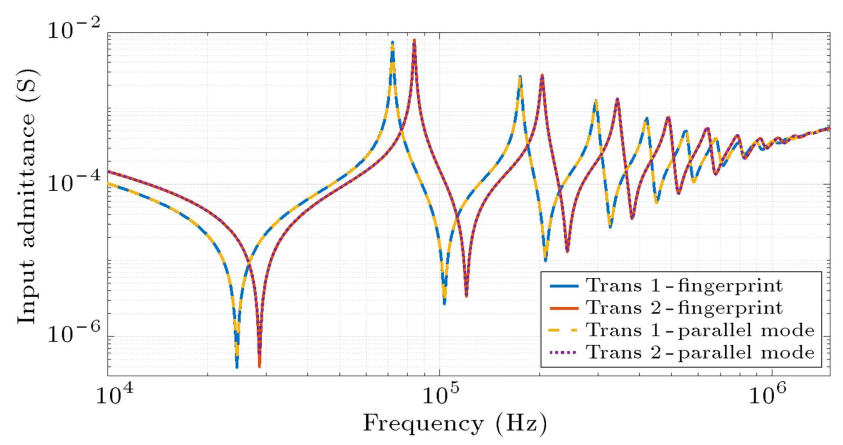

Figure 14. The frequency response of two parallel transformers obtained in the online mode. As can be seen, the online and offline transfer functions are identical.

impulse according to IEC 60060 strikes the line and initiates the transient signal. Figure 13 shows the described arrangement. As can be seen, the input current and voltage of the $\mathrm{HV}$ winding are measured for calculating the desired transfer functions.

The frequency responses due to the transient signals of lightning are shown in Figure 14. These responses are obtained using the STFT method. The other two methods are not shown since the previous section shows their weakness. It is apparent from Figure 14 that the frequency responses of these two transformers are not affected by each other. In fact, using high-frequency transients as the excitation signal makes the power system ineffective in the transfer function.

Several simulations like short circuit faults and lightning impulse striking in different locations of the network are studied. Based on these simulations, it is concluded that the presence of a parallel transformer or a transmission line may change the transient signals on the transformer; however, as long as the desired frequency components are excited, the frequency response of the transformer does not change. Even if these frequency components are filtered by the transmission line, the overall trace of the frequency response does not experience any major change and only fluctuates in higher frequencies. For instance, Figure 15 shows the frequency response of these two

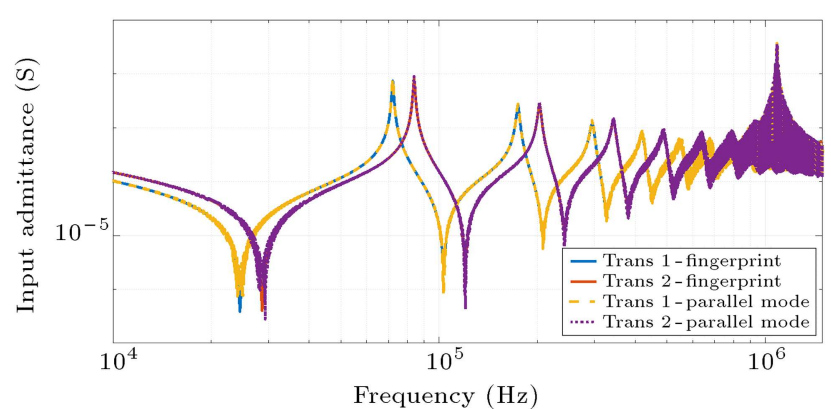

Figure 15. The frequency response traces of two parallel transformers obtained from the short circuit transient.

transformers obtained from a short circuit transient. These traces are extracted by the STFT method. As can be seen, the trances fluctuate in the higher frequency region. However, the extracted frequency response of each transformer is still in good agreement with the SFRA trace of that transformer. Figure 15 also shows the potential of the short circuit transients to be used for obtaining the online transfer function of the transformer.

\section{Conclusion}

Different mathematical methods for obtaining the transfer function of a transformer in online FRA were compared in this paper. The investigated method includes FFT, STFT, and the wavelet transform. In all cases, the SFRA was considered as the reference for choosing a suitable method. The comparison was performed on an appropriate model of the transformer, describing $\mathrm{LV}$ and $\mathrm{HV}$ windings and their electrical characteristics. Based on the presented results, it was concluded that the STFT method produced the most similar response compared with the SFRA trace. Moreover, this method worked satisfactorily in both current and voltage responses without any need for filtering the $50 \mathrm{~Hz}$ signal.

In the next step, the effect of other power network equipment on the online transfer function of the transformer was studied. The results revealed that the presence of a parallel transformer or a transmission line 
might change the transient waveform of the network; however, as long as the desired frequency components were excited, the measured frequency response of the transformer did not change. Studying the short circuit transient along with the lightning stroke indicated that using high-frequency transients as the excitation signal made the power system ineffective in the online transfer function.

\section{References}

1. Bagheri, M., Naderi, M.S., and Blackburn, T. "Advanced transformer winding deformation diagnosis: moving from off-line to on-line", IEEE Transactions on Dielectrics and Electrical Insulation, 19(6), pp. 18601870 (2012).

2. Su, C.Q. "Case study: lessons learned from the failure of a new $230-\mathrm{kV}$ transformer-cable termination", IEEE Electrical Insulation Magazine, 26(1), pp. 15-19 (2010).

3. Tenbohlen, S., Coenen, S., Djamali, M., et al. "Diagnostic measurements for power transformers", Energies, 9(5), p. 347 (2016).

4. Samimi, M.H., Akmal, A.A.S., Mohseni, H., et al. "Open-core optical current transducer: modeling and experiment", IEEE Transactions on Power Delivery, 31(5), pp. 2028-2035 (2016).

5. Gómez-Luna, E., Mayor, G.A., Guerra, J.P., et al. "Application of wavelet transform to obtain the frequency response of a transformer from transient signals-Part 1: theoretical analysis", IEEE Transactions on Power Delivery, 28(3), pp. 1709-1714 (2013).

6. Gomez-Luna, E., Mayor, G.A., Gonzalez-Garcia, C., et al. "Current status and future trends in frequencyresponse analysis with a transformer in service", IEEE Transactions on Power Delivery, 28(2), pp. 1024-1031 (2013).

7. Yao, C. "Transformer winding deformation diagnostic system using online high frequency signal injection by capacitive coupling", IEEE Transactions on Dielectrics and Electrical Insulation, 21(4), pp. 14861492 (2014).

8. Abu-Siada, A. and Islam, S. "A novel online technique to detect power transformer winding faults", IEEE Transactions on Power Delivery, 27(2), pp. 849-857 (2012).

9. Samimi, M.H., Tenbohlen, S., Akmal, A.A.S., et al. "Dismissing uncertainties in the FRA interpretation", IEEE Transactions on Power Delivery, 33(4), pp. 2041-2043 (2018).

10. Wang, M., Vandermaar, A.J., and Srivastava, K.D. "Review of condition assessment of power transformers in service", IEEE Electrical Insulation Magazine, 18(6), pp. 12-25 (2002).
11. Tenbohlen, S. "Experienced-based evaluation of economic benefits of on-line monitoring systems for power transformers", Cigré Session 2002, pp. 12-110 (2002).

12. Zhao, X., Yao, C., Zhao, Z., et al. "Performance evaluation of online transformer internal fault detection based on transient overvoltage signals", IEEE Transactions on Dielectrics and Electrical Insulation, 24(6), pp. 3906-3915 (2017).

13. Zhao, Z., Yao, C., Yan, M., et al. "Application of short time Fourier transform to obtain impulse frequency response curves of transformer winding deformation", in 2015 IEEE Conference on Electrical Insulation and Dielectric Phenomena (CEIDP), pp. 314-317 (2015).

14. Gomez-Luna, E., Silva, D., Aponte, G., et al. "Obtaining the electrical impedance using wavelet transform from the time response", IEEE Transactions on Power Delivery, 28(2), pp. 1242-1244 (2013).

15. Rybel, T.D., Singh, A., Vandermaar, J.A., et al. "Apparatus for online power transformer winding monitoring using bushing tap injection", IEEE Transactions on Power Delivery, 24(3), pp. 996-1003 (2009).

16. Behjat, V., Vahedi, A., Setayeshmehr, A., et al. "Diagnosing shorted turns on the windings of power transformers based upon online FRA using capacitive and inductive couplings", IEEE Transactions on Power Delivery, 26(4), pp. 2123-2133 (2011).

17. Zhao, Z., Yao, C., Zhao, X., et al. "Impact of capacitive coupling circuit on online impulse frequency response of a power transformer", IEEE Transactions on Dielectrics and Electrical Insulation, 23(3), pp. 12851293 (2016).

18. Tarimoradi, H. and Gharehpetian, G.B. "Novel calculation method of indices to improve classification of transformer winding fault type, location, and extent", IEEE Transactions on Industrial Informatics, 13(4), pp. 1531-1540 (2017).

19. Samimi, M.H., Tenbohlen, S., Akmal, A.A.S., et al. "Effect of different connection schemes, terminating resistors and measurement impedances on the sensitivity of the FRA method", IEEE Transactions on Power Delivery, 32(4), pp. 1713-1720 (2017).

20. Samimi, M.H. and Tenbohlen, S. "Using the temperature dependency of the FRA to evaluate the pressure of the transformer press ring", IEEE Transactions on Power Delivery, 33(4), pp. 2050-2052 (2018).

21. Rahimpour, E., Christian, J., Feser, K., et al. "Transfer function method to diagnose axial displacement and radial deformation of transformer windings", IEEE Transactions on Power Delivery, 18(2), pp. 493-505 (2003).

\section{Biographies}

Farzad Nasirpour received the BSc degree in Power Electrical Engineering from University of Sistan and Baluchestan in 2017. He is currently pursuing MSc degree in Electrical Engineering-Power Systems in 
University of Tehran. His research interests are highfrequency transformer modeling, asset management, and condition monitoring of power transformers.

Mohammad Hamed Samimi was born in Estahban, Iran in 1989. He received the BSc, MSc, and PhD degrees in Electrical Engineering from the University of Tehran, Iran in 2011, 2013, and 2017, respectively. $\mathrm{He}$ also won the DAAD scholarship award for the 'Binationality supervised doctoral degree' during $\mathrm{PhD}$ and was with the Stuttgart University for 20 months. He has been with the High Voltage Institute, University of Tehran as a Researcher and Assistant since 2009. He was with the Nirou Trans Company, Shiraz, working as a Researcher at the R\&D Department. He was also with the Iran Grid Management Company (IGMC), Tehran for two years as a researcher in the power system protection office. In 2018, he joined the Faculty of Engineering, University of Tehran where he is currently working as an Assistant Professor. His principal research interests include the design, testing, and diagnosis of high-voltage apparatuses.

Hossein Mohseni was born in Tehran, Iran in 1942. He received the Dipl.-Ing. and Dr.-Tech. degrees from the Technical University of Graz, Austria in 1971 and 1975, respectively. He was a Testing and Research Engineer with the High Voltage Laboratory and the Transformer Research and Development Department, ELIN Union AG, Austria from 1971 to 1976 . In 1976, he joined the Faculty of Engineering, University of Tehran. He is currently a Professor of Electrical Engineering and teaches high-voltage insulation technology and transient in power system and apparatus with Tehran University, Tehran. He is also a Technical Consultant with the Iran Power Generation and Transmission Company. 